\title{
Nephrotic syndrome in infants and toddlers before and after introduction of the meningococcal $B$ vaccine programme in England: an ecological study.
}

\author{
Nick Andrews ${ }^{1}{ }^{*}$, Julia Stowe ${ }^{1}$, Elizabeth Miller ${ }^{2}$ \\ ${ }^{1}$ Public Health England, London, UK \\ ${ }^{2}$ London School of Hygiene and Tropical Medicine, London, UK \\ *corresponding author: Nick Andrews, Statistics, Modelling and Economics Dept, Public \\ Health England , London, UK. nick.andrews@phe.gov.uk
}

\begin{abstract}
A possible increased risk of nephrotic syndrome (NS) following a meningococal group B vaccination campaign was identified during active safety surveillance in a province in Quebec, Canada where 4 cases were reported from an exposed population of $\sim 49,0000$, a higher rate than in provinces not using the vaccine. Meningococcal B vaccine has been given routinely at 2, 4 and 12 months of age in the United Kingdom since September 2015. To investigate the Canadian signal we used English hospital admissions data from 2005 to 2019 in 2-23 month old children to determine whether the rate of NS changed following the introduction of the vaccine. The analysis was stratified by age 2-5 months, 6-11 months, $12-$ 17 months and 18-23 months. The results showed no evidence of an increased risk with incidence rate ratios between 0.81 (95\% confidence interval $0.56-1.19)$ for age 6-11 months and $1.18(0.84-1.66)$ for age $12-17$ months.
\end{abstract}

\section{Key Words}

meningococcal B vaccine; nephrotic syndrome; ecological; vaccine safety

\section{Introduction}

The multicomponent group B meningococcal vaccine (4CMenB, Bexsero GSK, Rixensart, Belgium) was introduced in the United Kingdom (UK) in September 2015 as a 2,4,12 month $(2+1)$ schedule [1]. All birth cohorts from May 2015 were eligible with the first booster doses given from May 2016 as the first vaccine-eligible cohort reached 12 months of age. Vaccine uptake rapidly reached high levels with over $95 \%$ receiving the first dose and $87 \% 2+1$ doses. [2] By 2018 the vaccine had a clear impact on meningococcal group B disease in the target ages in England with a 75\% reduction compared to the expected incidence and with vaccine effectiveness of about $60 \%$ for the $2+1$ schedule. [2]

In clinical trials $4 \mathrm{CMenB}$ was found to be fairly reactogenic with fever within 6 hours of vaccination frequently reported [3] which was reduced by $40-50 \%$ if prophylactic 
paracetamol was given [4]. In Canada $4 \mathrm{CMenB}$ was used in a campaign beginning in May 2014 for children aged 2 months to 20 years in a region of Quebec where the incidence of meningococcal group B disease was high. Active post-vaccination safety follow-up was conducted by an online questionnaire. [5] The vaccine was given as four doses in infants under 6 months, three doses in older infants or two doses in those aged 1-20 years. The active surveillance produced a signal of a possible increased risk of nephrotic syndrome (NS), a renal disorder resulting in loss of albumen in the urine with generalised oedema in which relapse frequently occurs. Three cases of NS were identified by the active surveillance and an additional case found in a hospital database search. The cases occurred within 6 months of vaccination in children aged 2-5 years with the diagnoses made after a second dose (although two had symptoms prior to the second dose) and all were first episodes. Three cases were hospitalised giving a rate of 13.3 cases per 100,000 vaccinated individuals which was 3.6 fold (95\% CI 0.7-11.8) higher than in the rest of the province where the vaccine was not given, and 8.3 fold higher $(95 \% \mathrm{CI}$ : 1.1-62.0) higher than the rate in an 8 year pre-vaccination period.

An increased risk of NS has not been reported after other vaccines although a signal of an increased risk of NS relapse was raised with meningococcal $C$ conjugate vaccine [6].

However, a subsequent analytic epidemiological study found no evidence of an increased risk of NS relapse after the vaccine [7]. Given the signal from 4CMenB in Canada, and the extensive use of the vaccine in the UK, we conducted an ecological study to assess whether rates of NS changed following the mass introduction of the vaccine. Although the age range for $4 \mathrm{CMenB}$ in the UK is lower than that of the cases seen in Canada the large-scale use warrants the assessment of the NS signal. The high vaccine uptake and stable incidence of NS prior to vaccine introduction made an ecological study a feasible method to investigate the Canadian signal.

\section{Methods}

The Hospital Episodes Statistics (HES) dataset holds information on all hospital admissions to a National Health Service Hospital in England [8] and was used to identify NS cases.

Admissions with a diagnosis of nephrotic syndrome (ICD codes of N040 or N049) in the first diagnostic field were extracted for the period September 2005 to April 2019 for children aged 2-23 months of age. Any episode within 7 days of a previous episode was assumed to be part of the same episode. Episodes more than 7 days after a previous episode were assumed to be relapses. When assessing first episodes, to ensure all episodes were the first, only cases from September 2006-April 2019 were used for those aged 2-11 months and September 2007-April 2019 for those aged 12-23 months. Counts of cases were aggregated by the admission month and year and by the age of the case, and each of these cohorts assigned as vaccine eligible or not based on the corresponding birth cohort. Population data for England from 2005-2017 was obtained from the Office of National Statistics (ONS) to calculate person-time spent in each of these cohorts (population for 2018 was assumed to be the same as 2017) [9]. For descriptive analyses, counts and person time were then aggregated across age ranges of 2-5 months, 6-11 months, 12-17 months and 18-23 months as well as by year (September to 
August). Case numbers in each year were corrected to the 2017 population denominator for presentation. This meant for $2018 / 19$ case numbers were inflated to the expected number from a full year's follow-up rather than September -March. To compare the incidence before and after vaccination introduction, rates were calculated per 100,000 person years within the age groups and according to whether eligible for vaccination. Poisson regression was used to estimate the incidence rate ratio (IRR) in the vaccine eligible compared to non-eligible cohorts after checking for any secular trends. Analyses were done based on first episodes as well as using all episodes. Estimation of doses given to the population was from data on population coverage in the UK as used in the assessment of the impact of Meningococcal B vaccination. [2]

\section{Results}

A total of 876 episodes were obtained for 2005/06 to 2018/19 after removing those within 7 days. Of these, 328 were the first recorded admission in the period of which 9 were dropped in under one year olds for 2005/06 and 51 in one year olds for 2005-2007, as an earlier admission prior to these dates could not be excluded. Figures 1 and 2 show the adjusted counts over the period, with no evidence of an increase following the introduction of $4 \mathrm{CMenB}$ vaccination. NS admissions increased with age with the largest number of cases in the 18-23 month group. There was no evidence of an increasing trend in NS over time so unadjusted IRRs could be calculated. Tables 1 and 2 show the cases, person-time and incidence in the vaccine eligible and non-eligible periods by age group. The IRRs comparing vaccine eligible with non-eligible cohorts ranged from 0.81 to 1.18 with all $95 \%$ confidence intervals including one and the highest upper limit being 2.67 .

\section{Discussion}

In this follow-up period about 44 monthly birth cohorts were eligible for the first dose, 45 cohorts for the second and 34 for the booster dose of $4 \mathrm{CMenB}$. Based on vaccine coverage estimates of about $95 \%$ for the first dose, $93 \%$ for the second dose and $87 \%$ for the third dose [2] and an English birth cohort of about 55,000 per month this gives a total exposure of about 2.35 million first doses, 2.25 million second doses and 1.78 million booster doses. With this large number of doses administered in the period covered by this study it would be expected that an increased risk of NS of the size signalled in Canada, if it existed, would be detected. For example, if it is assumed that the risk is for at least 4-5 months post-vaccination, as suggested by the Canadian study, then in the vaccine eligible period much of the person-time in those aged 2-5 as well as 12-17 months will be post-vaccination risk time. If we assume about $80 \%$ of the $2-5$ month age group person time will be in the risk period (to allow for some delayed vaccination and 5\% unvaccinated) then to generate the observed upper end of the $95 \%$ CI for the IRR of 1.58 (for the cases including relapses) the true IRR would need to be $1.73(1.73 \times 0.8+1.00 \times 0.2=1.58)$. Similarly, if we assume about $65 \%$ of the $12-17$ month age period is risk (to allow for $80 \%$ coverage and some delayed vaccination) then to generate a IRR of 1.66 then the actual RI would need to be $2.0(2.00 \times 0.65+1.00 \times 0.35=1.66)$. 
Similar calculations based on first episodes (where upper 95\% CI IRRs were 2.14 and 1.91) would require the actual IRRs to be 2.43 for age 2-5 months and 2.40 for age 12-17 months.

This study has the usual limitations of an ecological study, with individual vaccination history not ascertained and inferences made about risk in individual vaccinees based on population aggregated data. However, with the high $4 \mathrm{CMenB}$ coverage levels in England a large increased risk should have been clear in these data. As shown by the above calculations, an increased risk of more than double after $4 \mathrm{CMenB}$ can be confidently excluded. While extraneous factors causing a reduction in NS incidence that happened to coincide with $4 \mathrm{CMenB}$ introduction could potentially have obscured a vaccine-associated risk, this seems unlikely given the stable incidence of NS over a long period prior to $4 \mathrm{CMenB}$ introduction. We also did not validate the NS cases by case note review. However, we did do case note reviews in our earlier HES based study of meningococcal $\mathrm{C}$ vaccine in which we used the same ICD codes to identify NS cases [7]. This showed that 53/64 (83\%) were confirmed demonstrating a high positive predictive value (PPV), although any PPV less than $100 \%$ would further slightly dilute a true risk. The study also does not address vaccination across a wide age range as in Canada for which the NS cases were in children vaccinated age 2-5 years, but does apply to the schedule as used in the UK with a much larger exposed population.

This ecological study therefore provides no evidence of an increased risk of NS after introduction of $4 \mathrm{CMenB}$ and provides evidence against IRRs of the size given as the point estimates in the study from Canada of 3.6-8.3.

Table 1: Comparison of the rate of nephrotic syndrome first episodes in the pre and post $4 \mathrm{CMenB}$ vaccination periods by age group.

\begin{tabular}{|l|l|l|l|l|l|}
\hline & \multicolumn{5}{|l|}{ Age of cases } \\
\hline Cohorts & & $2-5$ months & $6-11$ months & $12-17$ months & $18-23$ months \\
\hline \multirow{2}{*}{$\begin{array}{l}\text { Non- } \\
\text { vaccine } \\
\text { eligible }\end{array}$} & Cases & 27 & 19 & 41 & 122 \\
\cline { 2 - 6 } & Person years & 2012922 & 3144032 & 2988574 & 3154982 \\
\cline { 2 - 6 } & $\begin{array}{l}\text { Rate per } \\
100,000 \text { person } \\
\text { years }\end{array}$ & 1.34 & 0.60 & 1.37 & 3.87 \\
\hline \multirow{2}{*}{$\begin{array}{l}\text { Vaccine } \\
\text { eligible }\end{array}$} & Cases & 11 & 7 & 12 & 29 \\
\cline { 2 - 7 } & Person years & 771077 & 1031967 & 869423 & 703016 \\
\cline { 2 - 7 } & $\begin{array}{l}\text { Rate per } \\
100,000 \text { person } \\
\text { years }\end{array}$ & 1.43 & 0.68 & 1.38 & 4.13 \\
\hline \multirow{5}{*}{} & $\begin{array}{l}\text { Incidence rate } \\
\text { ratio } \\
(95 \% \text { CI) }\end{array}$ & $\begin{array}{l}1.06 \\
(0.53-2.14)\end{array}$ & $\begin{array}{l}1.12 \\
(0.47-2.67)\end{array}$ & $\begin{array}{l}1.01 \\
(0.53-1.91)\end{array}$ & $\begin{array}{l}1.07 \\
(0.71-1.60)\end{array}$ \\
\hline
\end{tabular}



Table 2: Comparison of the rate of nephrotic syndrome episodes including relapses in the pre and post $4 \mathrm{CMen} B$ vaccination periods by age group.

\begin{tabular}{|l|l|l|l|l|l|}
\hline & \multicolumn{5}{|l|}{ Age of cases } \\
\hline Cohorts & & $2-5$ months & $6-11$ months & $12-17$ months & $18-23$ months \\
\hline \multirow{2}{*}{$\begin{array}{l}\text { Non- } \\
\text { vaccine } \\
\text { eligible }\end{array}$} & Cases & 92 & 136 & 151 & 331 \\
\cline { 2 - 6 } & Person years & 2218743 & 3452763 & 3615307 & 3781714 \\
\cline { 2 - 6 } & $\begin{array}{l}\text { Rate per } \\
100,000 \text { person } \\
\text { years }\end{array}$ & 4.15 & 3.94 & 4.18 & 8.75 \\
\hline \multirow{2}{*}{$\begin{array}{l}\text { Vaccine } \\
\text { eligible }\end{array}$} & Cases & 34 & 33 & 43 & 56 \\
\cline { 2 - 7 } & Person years & 771077 & 1031967 & 869423 & 703016 \\
\cline { 2 - 7 } & $\begin{array}{l}\text { Rate per } \\
100,000 \text { person } \\
\text { years }\end{array}$ & 4.41 & 3.20 & 4.95 & 7.97 \\
\hline \multirow{5}{*}{} & $\begin{array}{l}\text { Incidence rate } \\
\text { ratio (95\% CI) }\end{array}$ & $\begin{array}{l}1.06 \\
(0.72-1.58)\end{array}$ & $\begin{array}{l}0.81 \\
(0.56-1.19)\end{array}$ & $\begin{array}{l}1.18 \\
(0.84-1.66)\end{array}$ & $\begin{array}{l}0.91 \\
(0.69-1.21)\end{array}$ \\
\hline
\end{tabular}


Figure1: Nephrotic syndrome first episode hospital admissions 2006/07-2018/19 by age group. Black bars indicate non-4CMenB eligible cohorts, dark grey partially eligible and light grey fully eligible. Case counts are corrected to the 2017/18 population denominator.
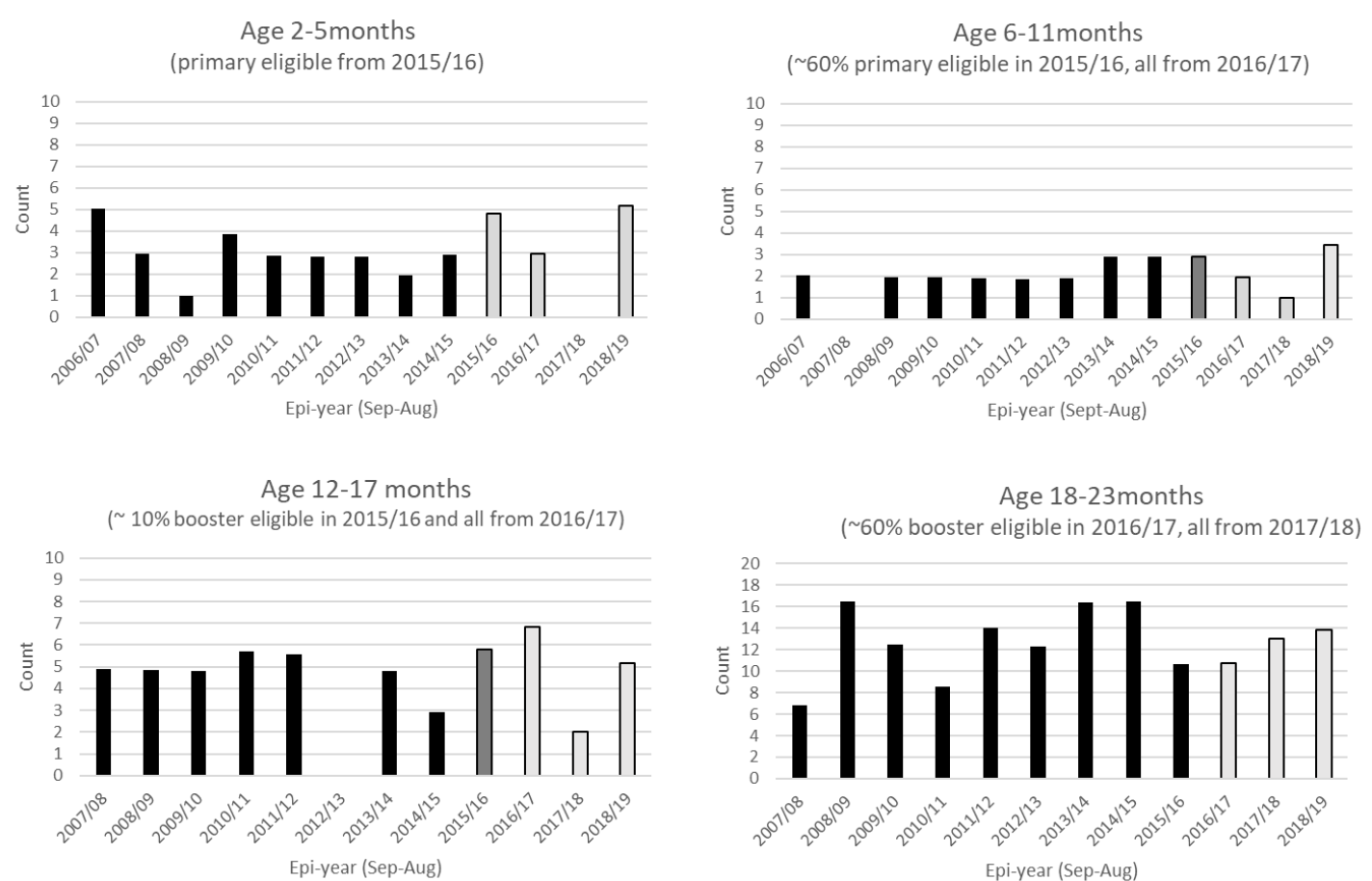

Figure 2: Nephrotic syndrome hospital admissions 2005/06-2018/19 by age group including relapses. Black bars indicate non-4CMenB eligible cohorts, dark grey partially eligible and light grey fully eligible. Case counts are corrected to the $2017 / 18$ population denominator.

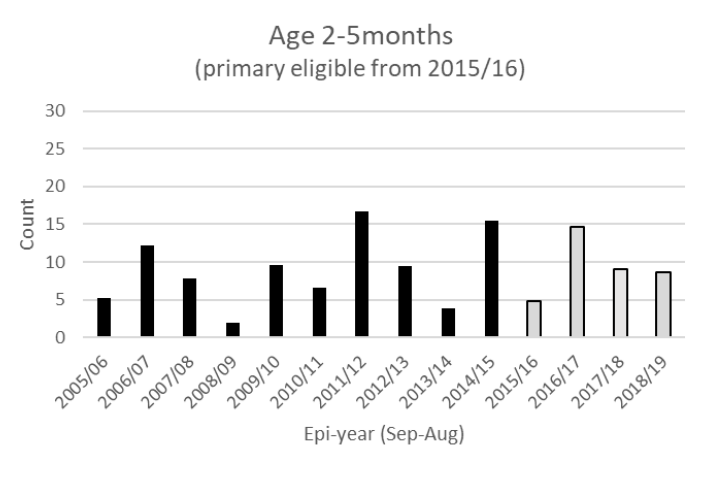

Age 12-17 months

( 10\% booster eligible in 2015/16 and all from 2016/17)

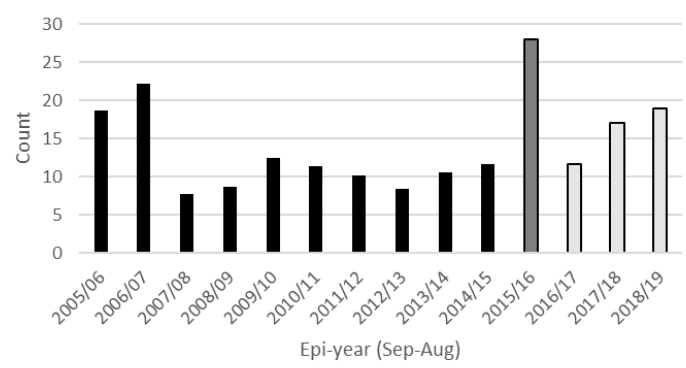

Age 6-11months

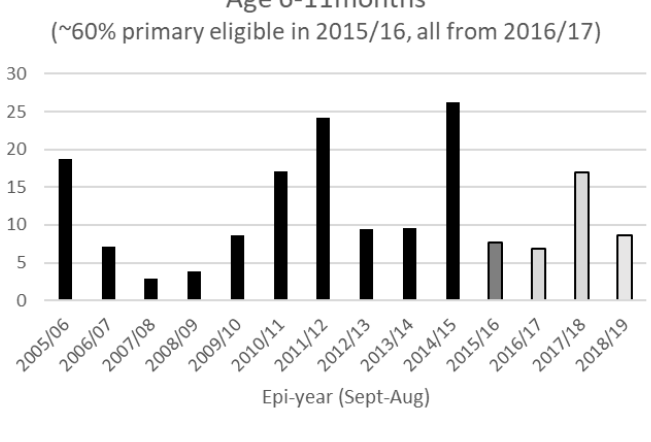

Age 18-23months

( $60 \%$ booster eligible in 2016/17, all from 2017/18)

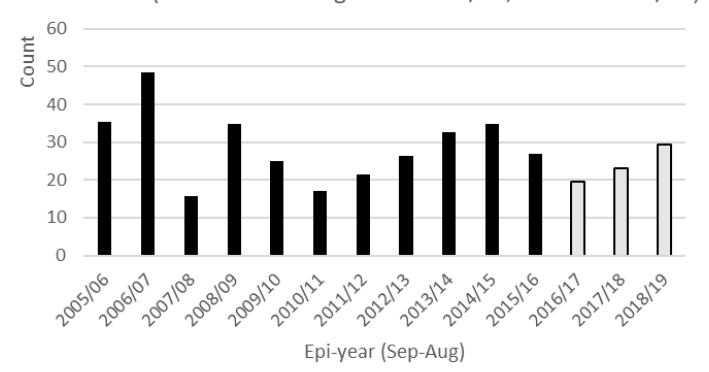




\section{Reference}

[1] Ladhani SN, Ramsay M, Borrow R, Riordan A, Watson JM, Pollard AJ. Enter B and W: two new meningococcal vaccine programmes launched. Arch Dis Child 2016; 101: 91-95.

[2] Ladhani SN, Andrews N, Parikh SR, Campbell H, White J, Edelstein M, Bai X, Lucidarme J, Borrow R, Ramsay ME. Vaccination of Infants with Meningococcal Group B Vaccine (4CMenB) in England. N Engl J Med. 2020;382(4):309-317.

[3] Vesikari T, Esposito S, Prymula R, et al. Immunogenicity and safety of an investigational multicomponent, recombinant, meningococcal serogroup B vaccine (4CMenB) administered concomitantly with routine infant and child vaccinations: results of two randomised trials. Lancet. 2013;381:825-35.

[4] Prymula R, Siegrist CA, Chlibek R, et al. Effect of prophylactic paracetamol administration at time of vaccination on febrile reactions and antibody responses in children: two open-label, randomised controlled trials. Lancet. 2009;374:1339-50.

[5] De Serres G, Billard MN, Gariépy MC, Roy MC, Boucher FD, Gagné H, Belley S, Toth E, Landry M, Skowronski DM. Nephrotic syndrome following four-component meningococcal B vaccination: Epidemiologic investigation of a surveillance signal. Vaccine. 2019;37(35):4996-5002.

[6] Abeyagunawardena AS, Goldblatt D, Andrews N, Trompeter RS. Risk of relapse after meningococcal C conjugate vaccine in nephrotic syndrome. Lancet. 2003;362(9382):449-50.

[7] Taylor B, Andrews N, Stowe J, Hamidi-Manesh L, Miller E. No increased risk of relapse after meningococcal $\mathrm{C}$ conjugate vaccine in nephrotic syndrome. Arch Dis Child. 2007;92(10):887-9.

[8] Hospital Episode Statistics (HES), Health and Social Care Information Centre. Copyright (C) 2020, Re-used with the permission of the Health and Social Care Information Centre. All rights reserved. http://www.hscic.gov.uk/hesdata (accessed 07/01/2020)

[9] Office for National Statistics. Population Estimates Unit. Estimated resident population mid year.

https://www.ons.gov.uk/peoplepopulationandcommunity/populationandmigration/population estimates (accessed 07/01/2020) 